\title{
A novel swine model for evaluation of dyslipidemia and atherosclerosis induced by human CETP overexpression
}

\author{
Tao Chen ${ }^{1,2}$, Meng Sun ${ }^{1,2}$, Jia-Qiang Wang ${ }^{3}$, Jin-Jin Cuil ${ }^{1,2}$, Zhong-Hua Liu ${ }^{3}$ and Bo Yu ${ }^{1,2^{*}}$
}

\begin{abstract}
Background: The mechanism of cholesteryl ester transfer protein (CETP) in lipid metabolism is still unclear. Furthermore, the relationship of CETP and atherosclerosis (AS) has been controversial. As pigs are a good model for both lipid and AS research, we investigated the lipid metabolism of human CETP (hCETP) transgenic pigs and explored the mechanism of CETP in lipid modulation.
\end{abstract}

Methods: Plasmids expressing the hCETP gene were designed, successfully constructed, and transfected into porcine fetal fibroblasts by liposomes. Using somatic cell nuclear transfer technology and embryonic transfer, hCETP transgenic pigs were generated. After the DNA, RNA, and protein levels were identified, positive $h C E T P$ transgenic pigs were selected. Blood samples were collected at different ages to evaluate the phenotypes of biochemical markers, and the metabolomes of plasma samples were analyzed by liquid mass spectrometry.

Results: Eight positive hCETP transgenic pigs and five negative cloned pigs were generated by transgenic technology. Finally, five hCETP transgenic and five cloned pigs were grown healthily. After feeding with a normal diet, hCETP transgenic pigs compared with unmodified pigs had no significant differences in body weight, liver function, kidney function, or plasma ions, while total cholesterol and low-density lipoprotein were higher than in unmodified pigs, and high-density lipoprotein was significantly decreased. Metabolomics analysis showed that there were differences in metabolic components between hCETP transgenic pigs, cloned pigs, and unmodified pigs.

Conclusions: In this study, we created hCETP transgenic pigs that could serve as an excellent model for lipid disorders and atherosclerosis.

Keywords: Cholesteryl ester transfer protein, Transgenic pig, Atherosclerosis, Metabolism

\section{Background}

Cholesteryl ester transfer protein (CETP) is a $74-\mathrm{KDa}$ hydrophobic glycoprotein that is secreted mainly from the liver and circulates in plasma. It is present in humans, rabbits, hamsters, chickens, and primates, but absent in rodents, pigs, cows, dogs, and horses [1]. CETP plays a critical role in lipid metabolism in humans, especially in mediating the exchange of cholesteryl esters (CE) and triglycerides (TG) between apoB-containing lipoproteins and high-density lipoprotein (HDL) [2]. The overall role

\footnotetext{
* Correspondence: yubodr@163.com

${ }^{1}$ The Key Laboratory of Myocardial Ischemia, Chinese Ministry of Education, Harbin, Heilongjiang, China

${ }^{2}$ Cardiology Division, The Second Affiliated Hospital of Harbin Medical University, No. 246 Xuefu Road, Harbin, Heilongjiang 150086, China Full list of author information is available at the end of the article
}

of CETP in atherosclerosis is complex, and whether CETP is an antiatherogenic or proatherogenic protein has been debated for many years. The results of introducing the CETP gene into rodents, which are naturally CETP-deficient, showed reduced HDL levels and severe atherosclerosis (AS) development [3-6]. Rabbits are highly susceptible to developing diet-induced AS because of their naturally high levels of CETP. In addition, it was reported that inhibiting CETP in a rabbit model of AS results in a marked reduction in AS [7]. Furthermore, genetic variants of CETP in humans with low CETP activity may be protective against cardio-vascular disease (CVD), since they exhibit higher HDL levels and reduced low-density lipoprotein (LDL) levels [8]. CETP inhibitors have been a hot topic of CVD research in 
recent years. To date, several CETP inhibitors have been brought to clinical trials. Although some CETP inhibitors have proven disappointing, some have been shown to induce significant changes in lipid profiles and metabolism, which permits some optimism for their role in cardiovascular risk reduction [9]. Therefore, more clinical and experimental data is needed to fully understand CETP and substances that affect it.

The majority of animal models used in research are rodents and rabbits whose physiological systems are significantly different from humans. Moreover, the small size of these animals precludes research in intravascular devices and is a challenge for noninvasive imaging. Many important progressive features of human atherosclerosis, such as plaque angiogenesis, plaque ruptures, and thrombosis, are rare or absent in rodent models [10]. Therefore, in recent years, transgenic pigs have been considered as a promising model for scientific research with the sequencing and annotation of the pig genome, given that their genome organization, anatomy, physiology, pathology, body weight, and lifespan closely resemble those of humans [11]. Because studies looking at the effects of CETP expression on lipid metabolism and atherosclerosis have produced contradictory results, generating a new transgenic pig model will be helpful for further CETP-related research.

In the present paper, we created such a genetic model by liver- and intestine-specific overexpression of the hCETP gene in pigs. As the pigs aged, this model developed increased plasma total cholesterol (TC) and LDL levels, and decreased plasma triglyceride (TG) and HDL levels. Metabolomics showed some key substances were associated with CETP function in lipid metabolism.

\section{Methods}

\section{Ethics statement}

All animal care and experiments in this research followed the guidelines of the Second Affiliated Hospital of Harbin Medical University and were approved by the Animal Use and Care committee. All animals (pigs) involved in this research were raised and bred following the guidelines of the Animal Husbandry Department of Heilongjiang, P.R. China.

\section{Genetic constructs}

The plasmid pEGFP-C1 (Clontech, CA, USA) was used as a skeleton. We directly subcloned human CETP, a 1.48-kb fragment with NheI/BamHI sites, from cDNA purchased from Sino Biological Inc. China (Catalog Number: HG13276-G). A 1.4-kb fragment of the human ApoC3 promoter was cloned from human genomic DNA with AseI/NheI sites as previously described [6]. The liverspecific expression promoter to drive the targeted $h C E T P$ gene and internal ribosome entry site (IRES) were inserted downstream of $h C E T P$, followed by enhanced green fluorescence protein $(E G F P)$ and an SV40 poly-A tail. Finally, the pApoCIII-hCETP-IRES-EGFP-SV40polyA gene construct was obtained whose structure is shown in Additional file 1: Figure S1-A. Primers used in PCR were as follows: human ApoC3 (Forward: 5' -ATTAA TATTCTGAGGGCAGAGCCG-3'; Reverse: 5' - GCT AGCCAGCTGCCTCTAGGG-3'); hCETP (Forward: 5'- GCTAGCATGCTGGCTGCCAC-3'; Reverse: 5' GGATCCCTAGCTCAAGCTCTGGAG-3').

\section{Generation of hCETP transgenic pigs}

Fibroblast cells derived from E32 fetuses were transfected by the liposome-mediated plasmid pApoC3hCETP-IRES-EGFP-SV40polyA, which was based on random insertion of nonhomologous DNA vector into the host genome. After G418 selection, surviving cells were propagated and confirmed by PCR as shown in Additional file 1: Figure S1-B; these were used as nuclear donors, and nuclear transfer was performed as previously described [12]. The reconstructed oocytes were activated and cultured for $18-22 \mathrm{~h}$, and the ones in a good growth state were surgically transferred into an oviduct of the surrogate. The surrogates were kept in a conventional environment for housing pigs. Pregnancies were confirmed by ultrasonography on day 28 , and all of the transgenic piglets were delivered by vaginal birth $24 \mathrm{~h}$ after induction with prostaglandin.

\section{Identification of hCETP transgenic pigs DNA analysis}

Each DNA sample was cleaved with EcoRI and NheI (TaKaRa, Dalian, China), which can digest the pig genome efficiently. Identification of $h C E T P$ transgenic pigs in DNA level was done using primers by PCR. The sequences of the primers were $5^{\prime}$-GAGCAAGGGCGAGGA GCTGTTCA-3' (forward) and 5' -TGCAGAATTCGAA GCTTGAGC-3' (reverse).

\section{Real-time PCR and western bolt analysis}

To examine the expression of $h C E T P$ in transgenic pig tissue, total RNA was isolated from 12 different tissues in two piglets. All samples were processed in triplicate, and the relative expression was standardized to GAPDH. Primers for the $h$ CETP gene were $5^{\prime}$ - CCTGACTGCT ACCTGTCTTTCCA-3' (forward) and 5' - TCCCTTCAG GACCAGCTTCAG-3' (reverse). Total proteins were isolated from plasma of pigs No. 1, 2, 3, 4, 5, 8, 10, 11, 12, 13 for detecting CETP expression. The detailed procedures of RT-PCR and western blotting were performed as previously described [12]. 


\section{Animals and diets}

The pigs were weaned at 28 days and fed a standard diet. All big white pigs were obtained and housed at the Northeast Agriculture University Research Institute (Harbin, China). Pigs had access to autoclaved water and normal diet (Animal husbandry of YuanDa, Harbin, China) ad libitum. Body weight was measured every month.

\section{Plasma analysis}

Blood samples were drawn from jugular veins into EDTA-coated tubes after an overnight fast at the time points indicated. Then they were stored on ice and centrifuged within $1.5 \mathrm{~h}$ at $1800 \mathrm{rpm}$ for $10 \mathrm{~min}$ at $4{ }^{\circ} \mathrm{C}$. All samples were stored at $-80{ }^{\circ} \mathrm{C}$ until analysis. Plasma TC, HDL, LDL, TG, glucose, liver function, and renal function were measured according to standard laboratory procedures of the Second Affiliated Hospital of Harbin Medical University.

\section{Metabolic analysis \\ Sample preparation}

Before RRLC-QTOF/MS analysis, the plasma and QC samples were thawed and refrozen in a $4{ }^{\circ} \mathrm{C}$ water bath. A volume of $1500 \mathrm{~mL}$ of methanol was added to $300 \mathrm{~mL}$ of plasma. After vortexing vigorously for $2 \mathrm{~min}$, the mixture was allowed to settle at $20{ }^{\circ} \mathrm{C}$ for $10 \mathrm{~min}$, and then centrifuged at $14,000 \mathrm{~g}$ for $15 \mathrm{~min}$ at $4{ }^{\circ} \mathrm{C}$. The supernatant was transferred to a clean vial and dried under nitrogen at $37{ }^{\circ} \mathrm{C}$. The residue was dissolved in $300 \mathrm{~mL}$ of acetonitrile-water $(3: 1, v / \mathrm{v})$, kept at $30{ }^{\circ} \mathrm{C}$ for $10 \mathrm{~min}$, and then vortex mixed for $60 \mathrm{~s}$. The supernatant was then placed into the sample vial for RRLC-QTOF/MS analysis [13].

\section{Liquid chromatography mass spectrometry}

A $10 \mathrm{~mL}$ aliquot of the pre-treated sample was injected into a $3.0100 \mathrm{~mm}(1.8 \mathrm{~mm})$ ZORBAX SB-C18 column (Agilent Technologies, Santa Clara, CA, USA) using a rapid resolution liquid chromatography system (1260 series, Agilent Technologies). All samples were maintained at $4{ }^{\circ} \mathrm{C}$ during the analysis [14]. Mass spectrometry was performed on an Agilent 6530-QTOF (Agilent Technologies) equipped with an electrospray ionization source operating in electrospray positive $\left(\mathrm{ESI}^{+}\right)$and electrospray negative $\left(\mathrm{ESI}^{-}\right)$modes. The detailed methods were as previously described [15].

\section{Statistical analysis}

The experimental data of each group were expressed as means \pm standard deviation. Student's $t$-test was applied to determine the differences between groups, and statistical significance was set at $P<0.05$. Statistical analysis was performed using SPSS13.0 software.

\section{Results}

Production of hCETP transgenic pigs

We obtained a total of 3 litters of pigs from 8 embryo transfers (as shown in Fig. 1a). In one litter, one female pig (No. 1) was born alive. In another litter, 9 female piglets (from No. 2 to No. 10) were born. However, 3 of them died due to deformities or developmental defects and 2 (No. 6 and No. 7) were so weak that they were euthanized to obtain tissues to measure the expression of CETP in vivo. In the last litter, 3 female piglets (No. 11 to No. 13) were born and thrived well.

\section{Identification of hCETP transgenic pigs}

Tissue samples of each piglet were taken and DNA was extracted, and the DNA level was identified by PCR. The results showed that pigs No. 2, 3, 5, 7, 9, 11, 12, and were positive $h C E T P$ transgenic pigs, and No. $1,4,6,8,10$, and 13 were negative cloned pigs (as shown in Fig. 1b).

RNA was extracted from 12 tissues of piglets No. 6 and No. 7 and detected by real-time PCR. The expression of $h C E T P$ was not detected in the liver of piglet No. 6 , while the liver of piglet No. 7 had high expression (Fig. 1c). The expression of $h C E T P$ in the tissues of piglet No. 7 (Fig. 1c) was consistent with theoretical levels, and $h C E T P$ expression in the liver and intestines of the piglets was the highest. The ApoC3 promoter successfully drove the specific expression of the target gene in the liver and intestine. Western blotting also identified that $h C E T P$ was expressed in the plasma of No. 2, 3, 5, 11, 12 (Fig. 1d).

\section{Animal characteristics}

The body weights of the $h C E T P$ transgenic and unmodified pigs showed an upward trend with age as shown in Additional file 1: Figure S1-C. However, there was no significant difference between $h C E T P$ transgenic pigs and unmodified pigs at each month of age $(P>0.05)$.

The plasma samples from $h C E T P$ transgenic, cloned, and unmodified pigs were subjected to analysis. Each group contained five pigs. The lipid profile is shown in Fig. 2a-d. At 2 months of age, the lipid profile was similar between $h C E T P$ transgenic and unmodified pigs, which showed no significant differences in plasma TC, TG, LDL, and HDL levels $(P>0.05)$. However, TC and LDL levels were increased in $h C E T P$ transgenic pigs compared with unmodified pigs at 5 months $(P<0.05)$. And HDL levels were decreased in transgenic pigs $(P<0.05)$. However, there was still no significant difference between these two groups in TG levels $(P>0.05)$.

Results showed that there were no significant differences in blood glucose levels of $h C E T P$ transgenic, cloned, and unmodified pigs $(P>0.05)$ (Fig. 2e). Moreover, liver and renal function of $h C E T P$ transgenic and cloned pigs were normal (Fig. 2f-i). This indicated that the process of human gene CETP integration into the 
$\mathbf{a}$

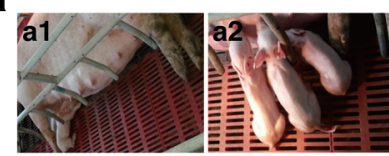

b

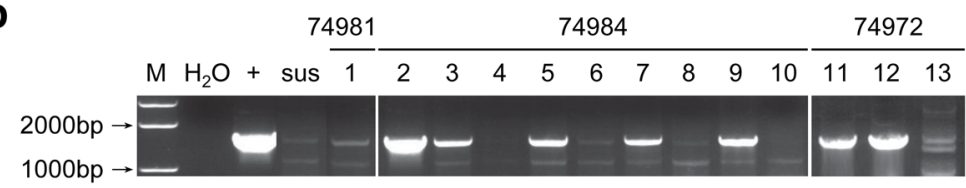

C

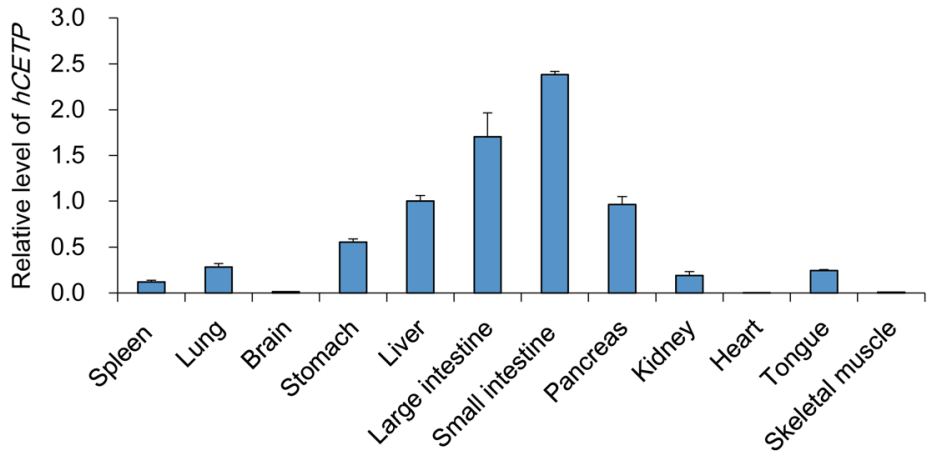

d

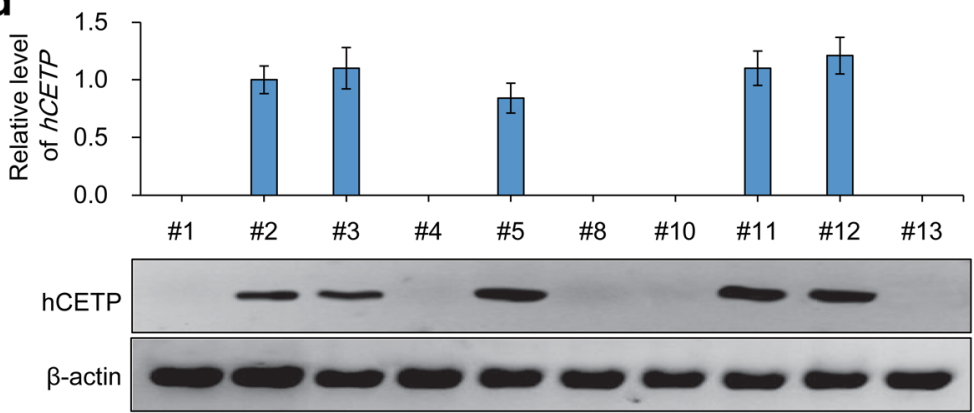

Fig. 1 Identification of hCETP transgenic pigs. a Identification results of 13 piglets by PCR. Piglets Nos. 2, 3, 5, 7, 9, 11, 12 were hCETP transgenic pigs. " + " indicates that the template is the pApoC3-hCETP-IRES-EGFP plasmid; "sus" indicates that the template is DNA from wild-type pig; " $\mathrm{H}_{2} \mathrm{O}$ "indicates that the template is water. b Generation of 13 piglets. B1: Piglet No. 1 born from sow No. 74981; B2: Piglets No. 11-13 born from sow No. 74972; B3: Piglets No. 2-10 born from sow No. 74984; B4: Piglet No. 6 was maldeveloped; B5: Piglet No. 7 was maldeveloped; c Expression pattern of hCETP in tissues of piglet No. 7; d: Western blot identification of CETP protein in piglets No. 1, 2, 3, 4, 5, 8, 10, 11, 12, 13

pig genome did not affect the metabolism of glucose or liver and renal function.

\section{Plasma metabolic profiling}

The RRLC-QTOF/MS chromatograms for hCETP transgenic, cloned, and unmodified pigs are shown in Fig. 3 and Additional file 1: Figure S2, which indicate peak distributions in positive mode $\left(\mathrm{ESI}^{+}\right)$and negative mode $\left(\mathrm{ESI}^{-}\right)$. From these two mode analyses, we found that these spectra include thousands of plasma metabolites, and there were overlapping peaks among the 3 groups. Moreover, excellent separations among these 3 groups were analyzed, and the results indicated that their plasma metabolites were significantly different from each other.
After pretreatment of the data by XCMS software, PCA analysis was used to observe the components classification trend of transgenic, cloned, and unmodified pigs in the $\mathrm{ESI}^{+}$mode. The classification effect of PCA worked well and showed that there were significant differences between genetically modified pigs (including $h C E T P$ transgenic pigs and negative cloned pigs) and unmodified pigs. However, this plot could not classify hCETP transgenic pigs and cloned pigs. PCA analysis correctly displayed the different components attribution in cloned and unmodified groups (Fig. 4a). Similar to ESI ${ }^{+}$mode, PCA analysis in $\mathrm{ESI}^{-}$mode could also distinguish the components of cloned and unmodified pigs (Fig. 4b).

In order to observe the classification between the two groups, we used the supervised learning method PLS-DA to further analyze the data. We chose the modeling component 


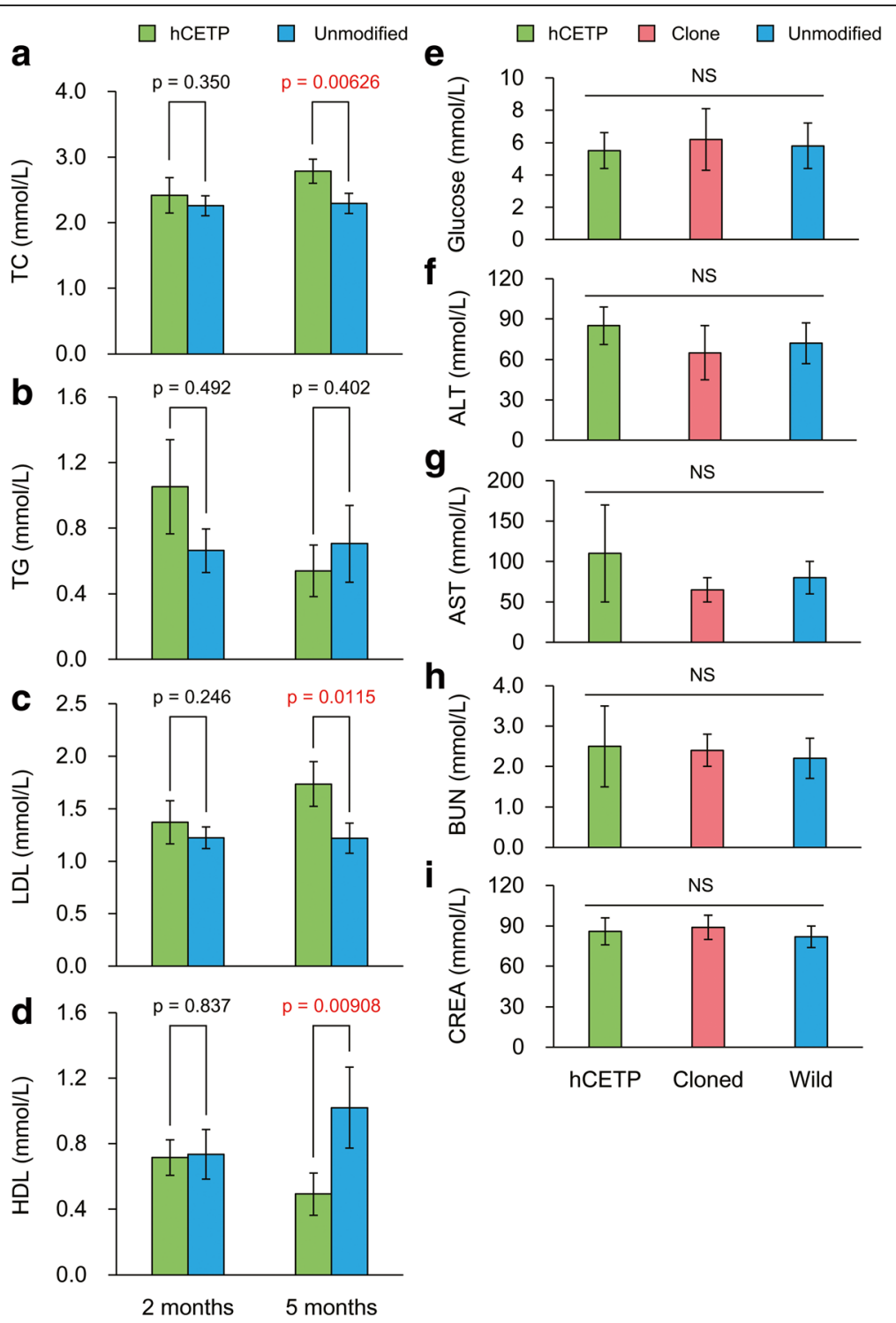

Fig. 2 Biochemical index changes in hCETP transgenic pigs. a TC: total cholesterol; b TG: triglyceride; c LDL: low density lipoprotein; $\mathbf{d}$ HDL: high density lipoprotein; e Glucose level at 5 months; f ALT (alanine transaminase) level at 5 months; $\mathbf{g}$ AST (glutamic-oxaloacetic transaminase) level at 5 months; $\mathbf{h}$ BUN (blood urea nitrogen) level at 5 months; i CREA (creatinine.) at 5 months

to construct the PLS score plot (as shown in Additional file 1: Figures S3 and S4). The results showed that the PLS-DA method could clearly show the classification of CETP transgenic, cloned, and unmodified pigs. There was no overlap among the 3 groups in the PLS-DA plot, which indicated that there was a significant difference between any two groups. For evaluating the effect of the PLS-DA model, we calculated $R^{2} X, R^{2} Y$, and $Q^{2}$. These parameters indicate the fit and prediction ability, respectively [16]. The results revealed that the PLS-DA model was valid.

\section{Discussion}

Whether results from rodent research can be directly translated into human clinical trials is still debated.
Large animal models that recapitulate human disease pathophysiology are thus attractive to researchers. The rapid development of genetic engineering technologies offers the possibility to do genetic modification in large animals. Here, we created $h C E T P$ transgenic pigs that overexpress CETP in a liver- and intestinespecific manner. In order to overexpress CETP specifically in liver and intestine, similar to the human expression profile, we chose the tissue-specific promoter ApoC3, which has been confirmed by Herrera et al. [6]. The present study using mass spectrometry metabolomics showed that the plasma metabolite profile of $h C E T P$ transgenic pigs differed from cloned pigs and unmodified pigs. 


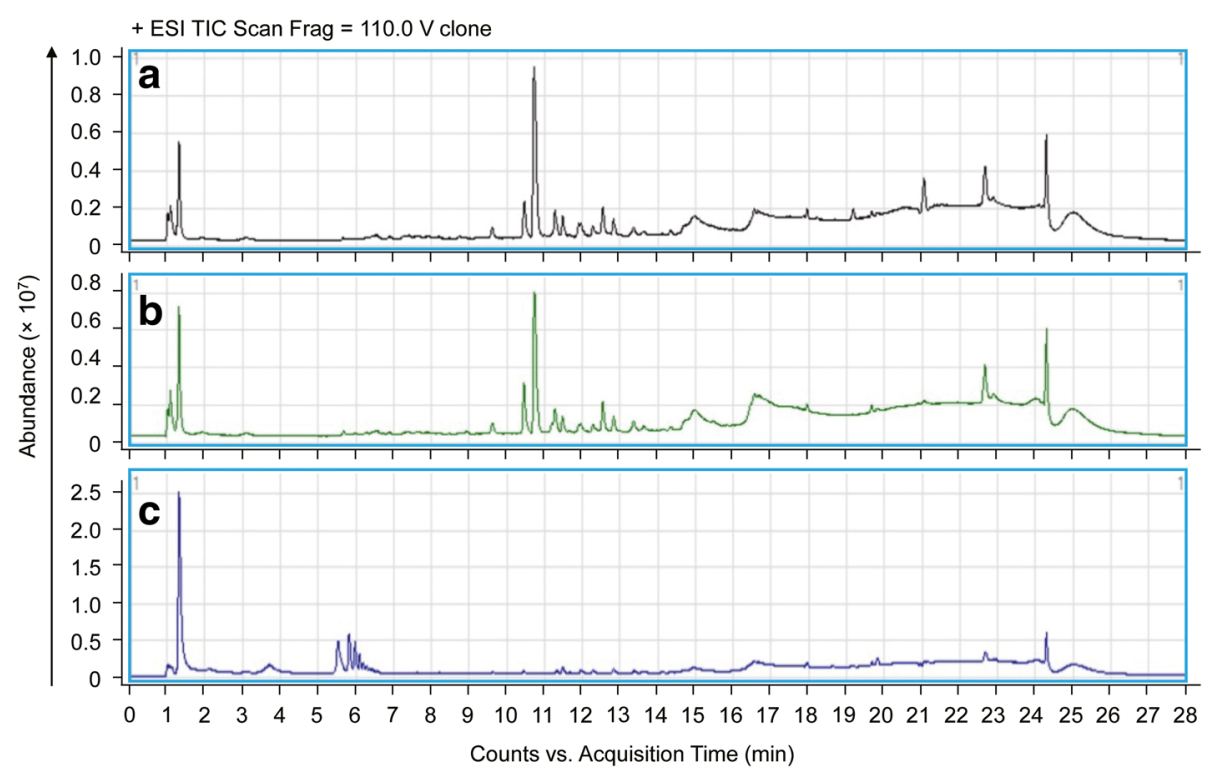

Fig. 3 Typical RRLC-QTOF/MS chromatograms of pig plasma samples acquired in positive mode. a cloned pig; b hCETP transgenic pig; c unmodified pig

It has been revealed that CETP plays a physiological role in modulating vascular lipoproteins. Previous human genetic research and animal studies have concluded that CETP is proatherogenic, and inhibiting its activity should reduce CVD risk [17]. However, some studies of genetic CETP deficiency do not show a strong association with CVD risk $[18,19]$. A genome-wide association study has identified 6 new loci associated with CVD, which included the CETP gene [20]. Our study also showed that CETP

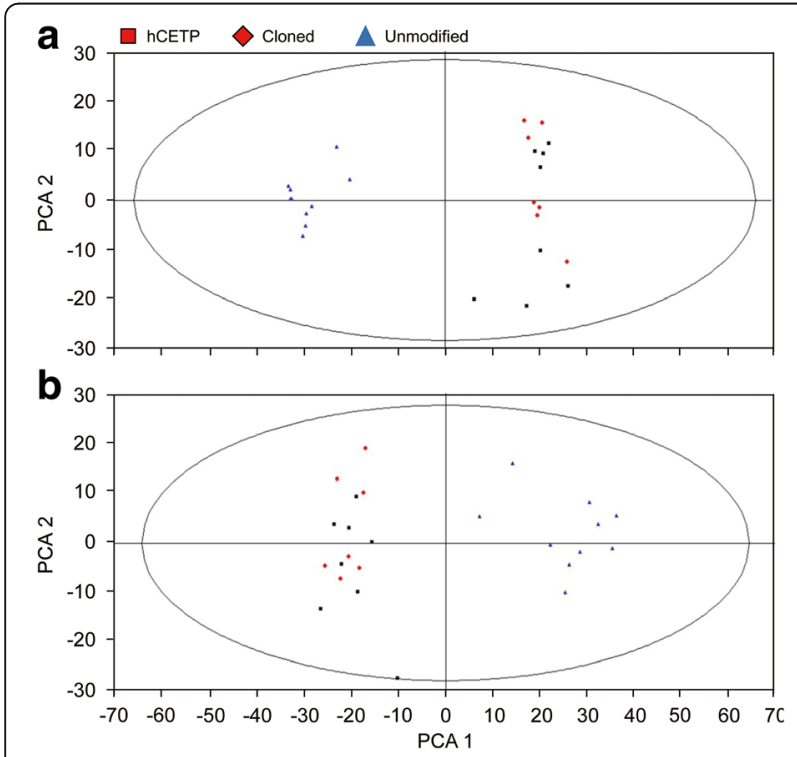

Fig. 4 PCA scores plot in different mode. a PCA scores plot for the top two components discriminated based on the data in $\mathrm{ESI}^{+}$mode. b PCA scores plot for the top two components discriminated based on the data in ESI ${ }^{-}$mode. $(\boldsymbol{\nabla})$ : hCETP transgenic pig; $(\square)$ : cloned pig; $(\mathbf{\Lambda})$ : unmodified pig should be proatherogenic, because along with the increase of age, TC and LDL levels of $h C E T P$ transgenic pigs were increased and significantly higher than those of normal pigs. However, changes of blood lipid levels in $h C E T P$ transgenic pigs were not as significant as those of CETP transgenic mice or rats $[3,6]$. One reason for this result may be the low copy number of foreign CETP genes integrating into the genomes of pigs due to using a plasmid vector by liposome transfection [21]. Another is that all generated $h C E T P$ transgenic pigs were heterozygotes produced by somatic cell nuclear transfer [22]. If homozygotes of $h C E T P$ transgenic pigs were generated after mating with each other, CETP gene expression could be enhanced. Pigs can develop AS under natural conditions, but require longer cycles. While high-fat and highcholesterol diet feeds can accelerate AS formation, the higher economic costs limit the application of this method $[23,24]$. In the last few decades, rabbits have been the main model of AS research for their extreme sensitivity to cholesterol, with CETP being highly expressed and AS plaques forming in a short time [25]. The greatest potential advances of genetically modified pigs in cardiovascular disease studies are that they could mimic human disorders and elucidate disease etiology. The heart, coronary vasculature, and blood flow of pigs is very similar to those of human beings, and overall, pigs can be used for drug, stent, or related interventional research, which can be detected by intracoronary imaging such as intravascular ultrasound (IVUS) or optical coherence tomography (OCT) [26-29]. The successful generation of hCETP transgenic pigs has laid the foundation for an excellent model to decipher the mechanisms of initiation and progression of AS. 
The metabolomics of transgenic pigs was analyzed by liquid phase mass spectrometry. After two methods of analysis, the results showed that $h C E T P$ transgenic positive, cloned, and unmodified pigs showed significant differences between the three metabolic components. First, a comparison of biochemical criteria in the three groups showed that $h C E T P$ transgenic pigs are different from cloned and control pigs, and there were no significant differences between cloned and unmodified pigs. However, the metabolic components between cloned pigs and unmodified pigs can be distinguished by PCA analysis, indicating that the metabolic components of the two are not identical. Somatic cell nuclear transfer technology requires that single somatic cell nuclei are introduced into enucleated oocytes containing some free DNA, which may have contributed to the variation. In addition, epigenetic modifications, intestinal bacteria, and intrauterine environmental factors may have an impact on the metabolic components of cloned pigs. Studies have also shown clear differences in phenotypic or metabolic components between cloned and normal animals [30-32].

The underlying mechanism by which CETP transfers lipoprotein is still not clearly understood, and the fundamental function of CETP in nature remains unknown $[9,33]$. The PLS-DA model not only distinguished the metabolic components of genetically modified pigs from unmodified pigs, but also showed discrepancy between transgenic positive and cloned pigs. The different metabolic components between hCETP transgenic and unmodified pigs might be closely associated with the physiological function of CETP, having effects on lipid metabolism, lipoprotein oxidation, inflammation, and fat synthesis [34]. Establishing the different metabolic components of hCETP transgenic positive, cloned, and unmodified pigs also needs further validation to identify the specific components, and then they can be analyzed in regards to possible mechanisms and related functions. The limitation of present study was the small sample size with only 5 pigs in each group, and a larger sample size would be more indicative of variation between animals in the multivariate analysis.

\section{Conclusions}

In conclusion, this report describes a model of lipid metabolic disturbance created by transgenesis in a large animal. The hCETP transgenic pigs showed hypercholesterolemia, and metabolomic analysis found CETP-related metabolic components. Therefore, this model should be valuable for further research into the mechanisms of CETP in the development of atherosclerosis.

\section{Additional file}

\begin{abstract}
Additional file 1: Figure S1. Eukaryotic expression vector construct and its expression in pig fibroblast cells. (A) Map of the 7728 bp plasmid. PApoC3: promotor specific expression in liver and intestine; hCETP: human CETP gene. IRES: internal ribosome entry sites; EGFP: enhanced green fluorescent protein. (B) Expression of pApoC3-hCETP-IRES-EGFP plasmid in pig fibroblast cells. "+": positive control; "S": transfected by plasmid of pig fibroblast cells; "-":negative control; " $\mathrm{H}_{2} \mathrm{O}^{\prime}$ : $\mathrm{H}_{2} \mathrm{O}$. C: Body weights of hCETP transgenic pigs. Figure S2. Typical RRLC-QTOF/MS chromatograms of pig plasma samples acquired in negative mode. (A) cloned pig; (B) hCETP transgenic pig; (C) unmodified pig. Figure S3. PLS-DA scores plot for components discriminated based on the data in positive mode. (A) transgenic pig and unmodified pig; (B) cloned and unmodified pig; (C) transgenic and cloned pig. (red square): positive pig; (red diamond): cloned pig; (blue triangle): unmodified pig. Figure S4. PLS-DA scores plot for components discriminated based on the data in negative mode. (A) transgenic pig and unmodfied pig; (B) cloned and unmodified pig; (C) transgenic and cloned pig. (red square): positive pig; (red diamond): cloned pig; (blue triangle): unmodified pig. (DOCX 1061 kb)
\end{abstract}

\section{Abbreviations \\ AS: Atherosclerosis; CE: Cholesteryl esters; CETP: Cholesteryl ester transfer protein; CVD: Cardio-vascular disease; HDL: High-density lipoprotein; \\ IVUS: Intravascular ultrasound; LDL: Low-density lipoprotein; OCT: Optical coherence tomography; TC: Total cholesterol; TG: Triglycerides}

\section{Acknowledgements}

Not applicable.

\section{Funding}

The project was financially supported by Innovation Fund of Harbin Medicine University (2016LCZX04).

\section{Availability of data and materials \\ All data generated or analyzed during this study are included within the article.}

\section{Authors' contributions}

Study design: TC, ZHL and BY. Study conduct: TC and MS. Data collection: JQW. Data analysis: JJC. Data interpretation: TC and BY. Drafting manuscript: TC. Revising manuscript content: BY. Approving final version of manuscript: TC, MS, JQW, JJC, ZHL and BY. TC takes responsibility for the integrity of the data analysis. All authors read and approved the final manuscript.

\section{Ethics approval}

All animal care and experiments in this research followed the guidelines of the Second Affiliated Hospital of Harbin Medical University and were approved by the Animal Use and Care committee. All animals (pigs) involved in this research were raised and bred following the guidelines of the Animal Husbandry Department of Heilongjiang, P.R. China.

\section{Consent for publication}

All data published here are under the consent for publication.

Competing interests

The authors declare that they have no competing interests.

\section{Publisher's Note}

Springer Nature remains neutral with regard to jurisdictional claims in published maps and institutional affiliations.

\section{Author details}

${ }^{1}$ The Key Laboratory of Myocardial Ischemia, Chinese Ministry of Education, Harbin, Heilongjiang, China. ${ }^{2}$ Cardiology Division, The Second Affiliated Hospital of Harbin Medical University, No. 246 Xuefu Road, Harbin, Heilongjiang 150086, China. ${ }^{3}$ College of life science, Northeast Agricultural University of China, Harbin, China. 
Received: 3 July 2017 Accepted: 4 September 2017

\section{Published online: 11 September 2017}

\section{References}

1. Barter PJ, Rye KA. Cholesteryl ester transfer protein inhibition as a strategy to reduce cardiovascular risk. J Lipid Res. 2012;53:1755-66.

2. de Grooth GJ, Klerkx AH, Stroes ES, Stalenhoef AF, Kastelein JJ, Kuivenhoven JA. A review of CETP and its relation to atherosclerosis. J Lipid Res. 2004;45: 1967-74.

3. Marotti KR, Castle CK, Boyle TP, Lin AH, Murray RW, Melchior GW. Severe atherosclerosis in transgenic mice expressing simian cholesteryl ester transfer protein. Nature. 1993;364:73-5.

4. Plump AS, Masucci-Magoulas L, Bruce C, Bisgaier CL, Breslow JL, Tall AR. Increased Atherosclerosis in ApoE and LDL Receptor Gene Knock-Out Mice as a Result of Human Cholesteryl Ester Transfer Protein Transgene Expression. Arterioscler Thromb Vasc Biol. 1999;19:1105-10.

5. Westerterp M, van der Hoogt CC, de Haan W, Offerman EH, Dallinga-Thie GM, Jukema JW, et al. Cholesteryl ester transfer protein decreases highdensity lipoprotein and severely aggravates atherosclerosis in $\mathrm{APOE}^{*} 3$ Leiden mice. Arterioscler Thromb Vasc Biol. 2006;26:2552-9.

6. Herrera VL, Makrides SC, Xie HX, Adari H, Krauss RM, Ryan US, et al. Spontaneous combined hyperlipidemia, coronary heart disease and decreased survival in Dahl salt-sensitive hypertensive rats transgenic for human cholesteryl ester transfer protein. Nat Med. 1999:5:1383-9.

7. Huang Z, Inazu A, Nohara A, Higashikata T, Mabuchi H. Cholesteryl ester transfer protein inhibitor (JT-705) and the development of atherosclerosis in rabbits with severe hypercholesterolaemia. Clin Sci (Lond). 2002;103:587-94.

8. Thompson A, Di Angelantonio E, Sarwar N, Erqou S, Saleheen D, Dullaart RP, et al. Association of cholesteryl ester transfer protein genotypes with CETP mass and activity, lipid levels, and coronary risk. JAMA. 2008;299:2777-88.

9. Filippatos TD, Klouras E, Barkas F, Elisaf M. Cholesteryl ester transfer protein inhibitors: challenges and perspectives. Expert Rev Cardiovasc Ther. 2016;14:953-62.

10. Bentzon JF, Falk E. Atherosclerotic lesions in mouse and man: is it the same disease? Curr Opin Lipidol. 2010;21:434-40.

11. Wernersson R, Schierup MH, Jorgensen FG, Gorodkin J, Panitz F, Staerfeldt $\mathrm{HH}$, et al. Pigs in sequence space: a 0.66X coverage pig genome survey based on shotgun sequencing. BMC Genomics. 2005;6:70

12. Kong Q, Wu M, Huan Y, Zhang L, Liu H, Bou G, et al. Transgene expression is associated with copy number and cytomegalovirus promoter methylation in transgenic pigs. PLoS One. 2009:4:e6679.

13. Bruce SJ, Tavazzi I, Parisod V, Rezzi S, Kochhar S, Guy PA. Investigation of human blood plasma sample preparation for performing metabolomics using ultrahigh performance liquid chromatography/mass spectrometry. Anal Chem. 2009;81:3285-96.

14. Dunn WB, Broadhurst D, Begley P, Zelena E, Francis-Mclntyre S, Anderson N, et al. Procedures for large-scale metabolic profiling of serum and plasma using gas chromatography and liquid chromatography coupled to mass spectrometry. Nat Protoc. 2011;6:1060-83.

15. Sun M, Gao X, Zhang D, Ke C, Hou Y, Fan L, et al. Identification of biomarkers for unstable angina by plasma metabolomic profiling. Mol BioSyst. 2013;9:3059-67.

16. Lin L, Huang Z, Gao Y, Yan X, Xing J, Hang W. LC-MS based serum metabonomic analysis for renal cell carcinoma diagnosis, staging, and biomarker discovery. J Proteome Res. 2011;10:1396-405.

17. Di Bartolo B, Takata K, Duong M, Nicholls SJ. CETP Inhibition in CVD Prevention: an Actual Appraisal. Curr Cardiol Rep. 2016;18:43.

18. Okamura T, Sekikawa A, Kadowaki T, El-Saed A, Abbott RD, Curb JD, et al. Cholesteryl ester transfer protein, coronary calcium, and intima-media thickness of the carotid artery in middle-age Japanese men. Am J Cardiol. 2009;104:818-22.

19. Regieli JJ, Jukema JW, Grobbee DE, Kastelein JJ, Kuivenhoven JA, Zwinderman $\mathrm{AH}$, et al. CETP genotype predicts increased mortality in statintreated men with proven cardiovascular disease: an adverse pharmacogenetic interaction. Eur Heart J. 2008;29:2792-9.

20. Webb TR, Erdmann J, Stirrups KE, Stitziel NO, Masca NG, Jansen H, et al. Systematic Evaluation of Pleiotropy Identifies 6 Further Loci Associated With Coronary Artery Disease. J Am Coll Cardiol. 2017;69:823-36.

21. Oliveira RR, Carvalho DM, Lisauskas S, Mello E, Vianna GR, Dode MA, et al. Effectiveness of liposomes to transfect livestock fibroblasts. Genet Mol Res. 2005:4:185-96.
22. Lagutina I, Fulka H, Lazzari G, Galli C. Interspecies somatic cell nuclear transfer: advancements and problems. Cell Reprogram. 2013;15:374-84.

23. Ratcliffe $H L$, Luginbuhl $H$. The domestic pig: a model for experimental atherosclerosis. Atherosclerosis. 1971;13:133-6.

24. Gerrity RG, Naito HK, Richardson M, Schwartz CJ. Dietary induced atherogenesis in swine. Morphology of the intima in prelesion stages. Am J Pathol. 1979;95:775-92.

25. Fan J, Kitajima S, Watanabe T, Xu J, Zhang J, Liu E, et al. Rabbit models for the study of human atherosclerosis: from pathophysiological mechanisms to translational medicine. Pharmacol Ther. 2015;146:104-19.

26. Ogita M, Miyauchi K, Onishi A, Tsuboi S, Wada H, Konishi H, et al. Development of Accelerated Coronary Atherosclerosis Model Using Low Density Lipoprotein Receptor Knock-Out Swine with Balloon Injury. PLoS One. 2016;11:e0163055.

27. Li Y, Fuchimoto D, Sudo M, Haruta H, Lin QF, Takayama T, et al. Development of Human-Like Advanced Coronary Plaques in Low-Density Lipoprotein Receptor Knockout Pigs and Justification for Statin Treatment Before Formation of Atherosclerotic Plaques. J Am Heart Assoc. 2016;5:e002779.

28. Iqbal J, Chamberlain J, Francis SE, Gunn J. Role of Animal Models in Coronary Stenting. Ann Biomed Eng. 2016;44:453-65.

29. Gongora CA, Shibuya M, Wessler JD, McGregor J, Tellez A, Cheng Y, et al. Impact of Paclitaxel Dose on Tissue Pharmacokinetics and Vascular Healing: A Comparative Drug-Coated Balloon Study in the Familial Hypercholesterolemic Swine Model of Superficial Femoral In-Stent Restenosis. JACC Cardiovasc Interv. 2015;8:1115-23.

30. Archer GS, Dindot S, Friend TH, Walker S, Zaunbrecher G, Lawhorn B, et al. Hierarchical phenotypic and epigenetic variation in cloned swine. Biol Reprod. 2003;69:430-6.

31. Landry AM, Landry DJ, Gentry LR, Green HL, Reggio B, Koonce KL, et al. Endocrine profiles and growth patterns of cloned goats. Cloning Stem Cells. 2005;7:214-25.

32. Clausen MR, Christensen KL, Hedemann MS, Liu Y, Purup S, Schmidt M, et al Metabolomic phenotyping of a cloned pig model. BMC Physiol. 2011;11:14.

33. Miller NE. Cholesteryl ester transfer protein: ace of spades, queen of hearts, or the joker? Front Pharmacol. 2015;6:145

34. Oliveira HC, de Faria EC. Cholesteryl ester transfer protein: the controversial relation to atherosclerosis and emerging new biological roles. IUBMB Life. $2011 ; 63: 248-57$

\section{Submit your next manuscript to BioMed Central and we will help you at every step:}

- We accept pre-submission inquiries

- Our selector tool helps you to find the most relevant journal

- We provide round the clock customer support

- Convenient online submission

- Thorough peer review

- Inclusion in PubMed and all major indexing services

- Maximum visibility for your research

Submit your manuscript at www.biomedcentral.com/submit
Biomed Central 